University of Nebraska - Lincoln

DigitalCommons@University of Nebraska - Lincoln

US Army Research

U.S. Department of Defense

2015

\title{
Advanced inductively coupled plasma etching processes for fabrication of resonator-quantum well infrared photodetector
}

J.Sun

U.S. Army Research Laboratory, Adelphi, MD

K. K. Choi

U.S. Army Research Laboratory, Adelphi, MD

M. D. Jhabvala

U.S. Army Research Laboratory, Adelphi, MD

C. A. Jhabvala

U.S. Army Research Laboratory, Adelphi, MD

A. Waczynski

U.S. Army Research Laboratory, Adelphi, MD

See next page for additional authors

Follow this and additional works at: http://digitalcommons.unl.edu/usarmyresearch

Sun, J.; Choi, K. K.; Jhabvala, M. D.; Jhabvala, C. A.; Waczynski, A.; and Olver, K., "Advanced inductively coupled plasma etching processes for fabrication of resonator-quantum well infrared photodetector" (2015). US Army Research. 330.

http://digitalcommons.unl.edu/usarmyresearch/330

This Article is brought to you for free and open access by the U.S. Department of Defense at DigitalCommons@University of Nebraska - Lincoln. It has been accepted for inclusion in US Army Research by an authorized administrator of DigitalCommons@University of Nebraska - Lincoln. 
Authors

J. Sun, K. K. Choi, M. D. Jhabvala, C. A. Jhabvala, A. Waczynski, and K. Olver 


\title{
Advanced inductively coupled plasma etching processes for fabrication of resonator-quantum well infrared photodetector
}

\author{
J. Sun *, K.K. Choi, M.D. Jhabvala, C.A. Jhabvala, A. Waczynski, K. Olver \\ U.S. Army Research Laboratory, Adelphi, MD 20783, USA \\ NASA Goddard Space Flight Center, Greenbelt, MD, USA
}

\section{H I G H L I G H T S}

- Resonator-quantum well infrared photodetectors (R-QWIPs) use resonances to increase the quantum efficiency (QE).

- The QEs observed is $70.8 \%$ in one of the test detectors.

- The test results agree with EM designs satisfactorily.

- The spectral uniformity of the FPA is about $3 \%$.

\section{A R T I C L E I N F O}

\section{Article history:}

Received 5 August 2014

Available online 28 September 2014

\section{Keywords:}

Inductively coupled plasma etching

Resonator-quantum well infrared

photodetectors focal plane array

GaAs substrate removal

\begin{abstract}
A B S T R A C T
Resonator-quantum well infrared photodetectors (R-QWIPs) are the next generation of QWIP detectors that use resonances to increase the quantum efficiency $(\mathrm{QE})$. To achieve the expected performance, the detector geometry must be produced in precise specification. In particular, the height of the diffractive elements (DE) and the thickness of the active resonator must be uniformly and accurately realized to within $0.05 \mu \mathrm{m}$ accuracy and the substrates of the detectors have to be removed totally. To achieve these specifications, two optimized inductively coupled plasma (ICP) etching processes are developed. Using these etching techniques, we have fabricated a number of R-QWIP test detectors and FPAs with the required dimensions and completely removed the substrates of the test detectors and FPAs. Their QE spectra were tested to be in close agreement with the theoretical predictions. The operability and spectral non-uniformity of the FPA is about $99.57 \%$ and $3 \%$ respectively.
\end{abstract}

Published by Elsevier B.V.

\section{Introduction}

QWIP holds many advantages over other detector FPAs, such as inexpensive and large area substrates, simple material design and growth, effective self-passivation, absence of $1 / f$ noise, and high FPA resolution, sensitivity, uniformity and operability [1]. However, a typical $5 \%$ quantum efficiency (QE), which is defined as the fraction of external incident light absorbed per pixel area, in QWIP focal plane arrays (FPAs) requires the detectors to operate at a long integration time of $5 \mathrm{~ms}$ or longer. This long integration time has prevented their applications in high speed imaging $[1,2]$. Recently, we have established a three-dimensional finite element electromagnetic (EM) model to calculate QE quantitatively [3-6]. This theoretical tool allows us to design new optical

\footnotetext{
* Corresponding author at: U.S. Army Research Laboratory, Adelphi, MD 20783,
} USA. coupling structures to achieve a larger QE. One of these detector structures is known as the resonator-QWIP or R-QWIP.

An R-QWIP consists of a properly sized detector pixel volume. On the top of the pixel, there is an array of diffractive elements (DEs) that are made of the same GaAs contact material and are covered with an ohmic metal layer and a gold layer, which are shown in Fig. 1(A). In the bottom of the pixel, there is a common ground contact layer, and the substrate underneath is completely removed. The light is incident from the bottom and is scattered by the DEs back to the detector volume. Aided by the EM model, we adjusted the dimensions of the DEs and the active volume such that the scattered light circulates inside the detector resonantly, with which the internal optical intensity can be greatly increased. The higher intensity leads to larger QEs and faster detection speeds.

To achieve the expected resonances, the substrate has to be removed to prevent the escape of unabsorbed light from the detector. After scattered by the DEs, the light usually travels at an angle larger than the critical angle for total internal reflection at the 
bottom semiconductor/air interface. Without the substrate, the light can then be reflected back by the bottom layer and still stays inside the same detector. Therefore, removing the substrate is an essential step in producing an R-QWIP. Since this detector relies on constructive interference between the incident light and the reflected light, the designed detector dimensions have to be produced accurately. To ensure proper phase relationship for constructive interference, the active material thickness and the height of the DEs have to be fabricated to within $0.05 \mu \mathrm{m}$ of the EM design specification in order to yield a QE that stays within $10 \%$ of the designed value.

To achieve this accuracy, we applied two optimized inductively coupled plasma (ICP) etching processes (selective and non-selective) to fabricate the test devices and FPAs. In an ICP system, there are two independent plasma power sources that provide almost independent control of ion density and ion energy. Ion density is controlled by ICP source power alone. Increasing ICP source power increases ion density. Meanwhile, ion energy is affected by both ICP source power and RF chuck power. Increasing ICP power decreases induced dc bias. On the contrary, increasing RF chuck power raises dc bias on the chuck. Since an ICP system provides one more process parameter than a reactive ion etching (RIE) system for plasma control, it is more flexible to use ICP to optimize different etching processes, such as selective (etching GaAs over $\mathrm{Al}_{x} \mathrm{Ga}_{1-x}$ As stop etching layer) versus non-selective etching, or isotropic versus vertical etching [7-10].

In addition to the etching profile, the electrical damage to the detectors produced by the active gases in the plasma is also an important consideration. There are many physical and chemical processes involved in the plasma etching. A general conclusion attributes the main plasma damage to the high energy ions via physical bombardment and the reactive ions via chemical reactions [7,9-12]. The use of a low RF chuck power in ICP will reduce the ion energy; and the use of a chlorine-based gas will eliminate the harmful chemical reactions present in the hydrogen-based gas.

Previously, we had optimized a selective etching process that could yield a very high selectivity $(>5000: 1)$ and a fast GaAs etching rate $(2700 \AA / \mathrm{min})$ [13]. The etching surface was perfectly smooth and mirror-like after processing. In addition to its simplicity, the process is also highly reproducible and shows no damage to the detector material. In this work, we applied the same processes to fabricate the new detector structures. For test devices, each
R-QWIP detector is approximately $22 \times 22 \mu \mathrm{m}^{2}$ in size. To increase the signal to noise ratio, we grouped the detectors into arrays of $40 \times 40$ elements. The detectors in each array were connected in parallel using a fanout circuit. The pixel pitch is $25 \mu \mathrm{m}$. After we fabricated and test the test devices successfully, we subsequently produced several FPAs with $640 \times 512$ and $1 \mathrm{~K} \times 1 \mathrm{~K}$ formats. Fig. 1(A) shows the designed R-QWIP structure, and Fig. 1(B) shows material layer structure. The active $\mathrm{QW}$ layers typically consists of 21 periods of $48 \AA \mathrm{GaAs}$ and $500 \AA \mathrm{Al}_{0.242} \mathrm{Ga}_{0.758} \mathrm{As}$, doped to $0.8 \times 10^{18} \mathrm{~cm}^{-3}$.

\section{Fabrication of test device and FPAs}

Our test device and FPA fabrication process requires five masks. We used the first mask to create an array of rings as the DEs to diffract normal incident light into nearly parallel propagation. A standard photolithography process was utilized to pattern 4-in. wafers. $1.8 \mu \mathrm{m}$ thick AZ5214 photoresist was coated on the wafer by using an EVG 120 Resist Processing Cluster. The resist was baked at $120^{\circ} \mathrm{C}$ for one minute in the system. The DEs were formed by using our optimized selective ICP etching process to reach down to the $15 \AA$ top stop etching layer, which is shown in Fig. 1(B). The etching depth is $5000 \AA$. The optimized etching parameters were: $\mathrm{BCl}_{3}=20 \mathrm{sccm}, \mathrm{SF}_{6}=10 \mathrm{sccm}, \mathrm{Ar}=10 \mathrm{sccm}$; pressure = $0.5 \mathrm{mTorr}$; $\mathrm{RF}$ Power $=0 \mathrm{~W}$; ICP Power $=200 \mathrm{~W}$; and substrate temperature $=25^{\circ} \mathrm{C}$. Before the ICP etching, one minute oxygen plasma was used to clean the etching surface. The etching process was conducted in a Unaxis VLR 700 Etch System. The tool uses a $2 \mathrm{MHz}$ RF inductively coupled coil to generate high density plasma. Ion energy at the wafer surface is independently controlled by a $13.56 \mathrm{MHz} \mathrm{RF}$ bias applied to the cathode. Wafer temperature is maintained through the use of a fluid cooled cathode in conjunction with electrostatic clamping and helium backside cooling. A typical schematic of an ICP etching system can be found in Fig. 1 of Ref. [14]. Since the selective etching process has a very high selectivity (greater than 5000:1 for $\mathrm{Al}_{0.4} \mathrm{Ga}_{0.6} \mathrm{As}$ ), $15 \AA$ thick stop etching layer is sufficient to define the DE height. The stop etching layer is also important for etching uniformity across the wafer as the etching rate is higher near the wafer edge. Fig. 2 is a SEM image of the DEs. The etching is uniform, and the etching surface is clean and smooth.

The second masking step defines the ground contact area located outside the pixel area. Our non-selective ICP etching recipe
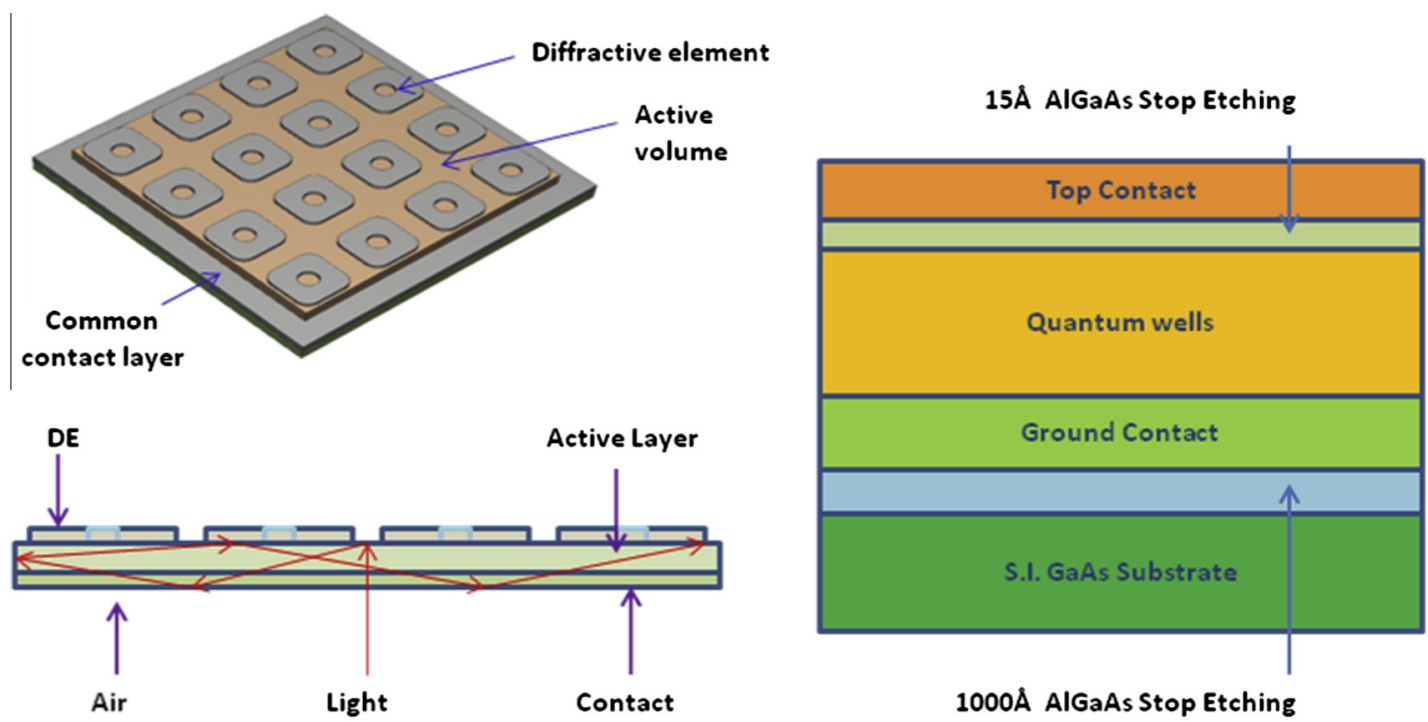

(A)

(B)

Fig. 1. The figure shows the designed R-QWIP structure (A) and material layer structure (B). 
was used to reach the common ground contact layer. The optimized etching parameters were: $\mathrm{BCl}_{3}=50 \mathrm{sccm}, \mathrm{Ar}=10 \mathrm{sccm}$; pressure $=5 \mathrm{mTorr}$; RF Power $=80 \mathrm{~W}$; ICP Power $=800 \mathrm{~W}$; and substrate temperature $=25^{\circ} \mathrm{C}$. In this step, a finite $R F$ power was necessary to create a vertical sidewall. We nevertheless minimized the power to avoid the possible plasma damage. The RF power needed for the creation of the plasma also induces a DC voltage on the chuck, which accelerates the ions toward the etching material. By using a low RF power and a high ICP power, the DC voltage on the chuck is only $120 \mathrm{~V}$. Therefore, the physical impact of these ions onto the detector material can be reduced. Fig. 3 was taken after ground contact Etching of a $640 \times 512$ FPA. The outside dark stripes are ground contact areas.

We used the third mask to define the lift-off areas for the deposition of $\operatorname{Pd}(50 \AA) / \mathrm{Ge}(200 \AA) / \mathrm{Au}(300 \AA) / \operatorname{Pd}(50 \AA) / \mathrm{Au}(5000 \AA)$ metal and it was followed by a furnace annealing at $350{ }^{\circ} \mathrm{C}$ for $25 \mathrm{~min}$. After this process, the diffractive elements (DEs) were covered with an ohmic metal layer and a gold layer. The gold layer is used to reflect the incident light and DEs are used to convert the polarization of the incident light from horizontal to vertical through diffraction. Fig. 4 shows one corner of the test device after metallization. The inside square is the $40 \times 40$ test detector pixel area.

We used the fourth mask to define the pixels. We opened the pixel areas while other areas were covered with photoresist. In the pixel areas, the metal squares were used as etching masks and non-selective ICP etching was utilized to create individual pixels. Fig. 5 shows microscope pictures taken after the pixel ICP etching. The fifth mask is indium bump mask. We coated $10 \mu \mathrm{m}-$ thick positive photoresist (AZ9245) and deposited $6 \mu \mathrm{m}$-tall indium bumps on the wafer using a thermal evaporator. The wafer was diced into test devices and FPAs. The candidates were bump bonded to the high resistivity silicon circuit boards and hybridized to readout integrated circuits. Then the backsides of the test detectors and FPAs were filled with low viscosity epoxy.

\section{Substrate removal of test devices and FPAs}

Thinned QWIP FPAs offers several advantages over unthinned FPAs. First, the thermal mass of the FPAs is reduced to lessen the detector cooling time. The thinned layer also makes it easier to adapt to the thermal expansion mismatch between GaAs and the silicon readout circuit. Optical crosstalk among pixels is suppressed by better optical confinement. Besides these general benefits, substrate removal is specifically required for R-QWIP. The thinned R-QWIP FPAs enhance the resonant effects, and the QE can increase by a factor of 3-4 according to EM modeling.

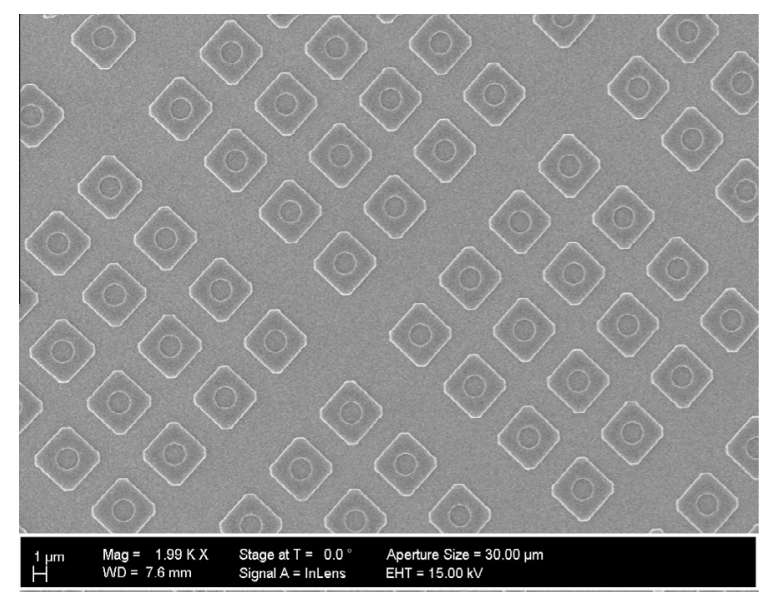

Fig. 2. A SEM image taken after first ICP etching.

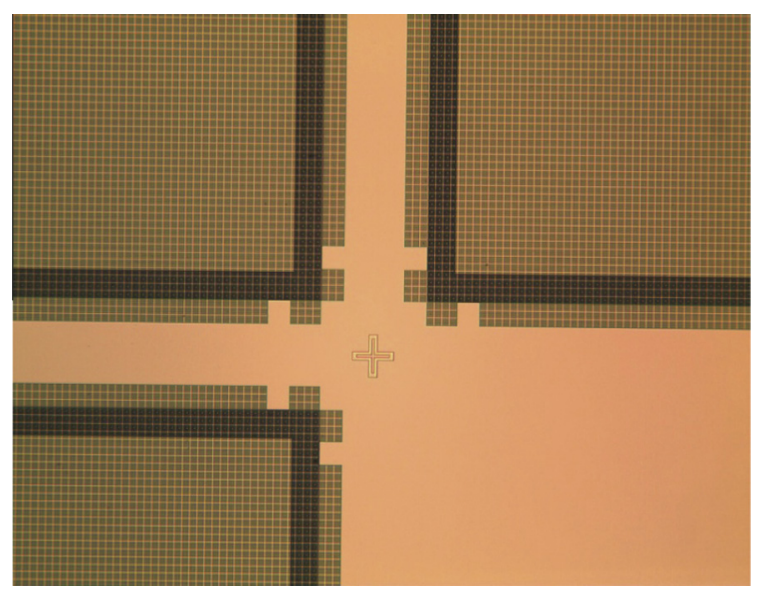

Fig. 3. A microscope picture taken after ground contact Etching $(640 \times 512 \mathrm{FPA})$.

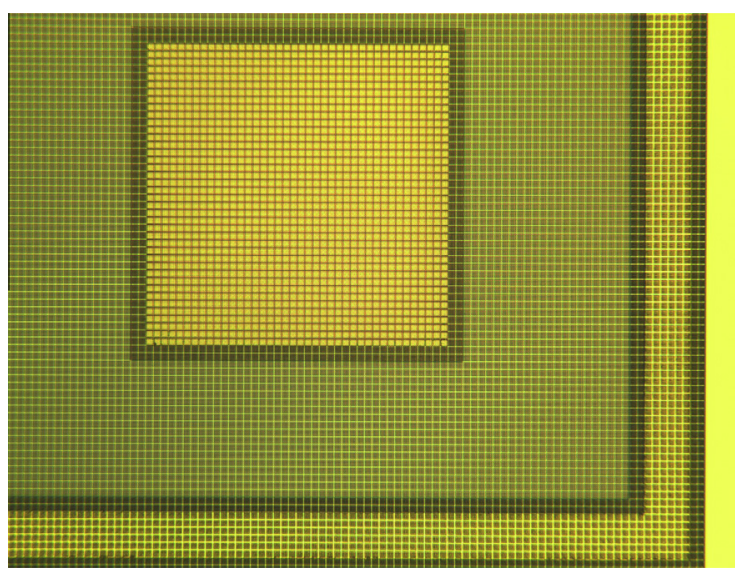

Fig. 4. A microscope picture taken after metallization.

The substrate removal process includes two steps, which are mechanical lapping and selective ICP etching. The substrates of the test device and FPAs were first mechanically lapped, using $3 \mu \mathrm{m}$ calcined aluminum oxide lapping medium, to within $50 \mu \mathrm{m}$. The edges of the test devices and FPAs were then hand painted with a surface coating. After baking the test devices and FPAs in an oven for one hour at $95^{\circ} \mathrm{C}$, the test devices and FPAs were introduced into the Unaxis VLR 700 Etch System for about two hour of etching. After ICP etching, solvents and Q tips were used to clean etching surface and remove the surface coating on backside and the edges of the FPAs. Fig. 6 shows two pictures, one was taken after lapping but before ICP etching, and another was taken after etching. As seen in these pictures, the surface of the die is uniform, smooth and mirror-like after etching. The etching surface is close enough to the pixels such that the pixels on the other side can be seen under microscope.

\section{Test results of test devices and FPAs}

Although we optimized the DE design, due to the developmental nature of our detector processing, we obtained different DE patterns as shown in Fig. 5. In addition, the thicknesses of the DE layer and the quantum well active layer are also different. As a result, different QEs were obtained for the two detectors. We measured the conversion efficiency (CE) spectrum for each R-QWIP and its 


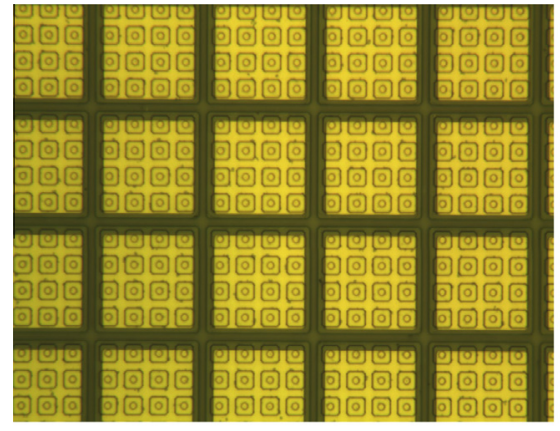

(A)

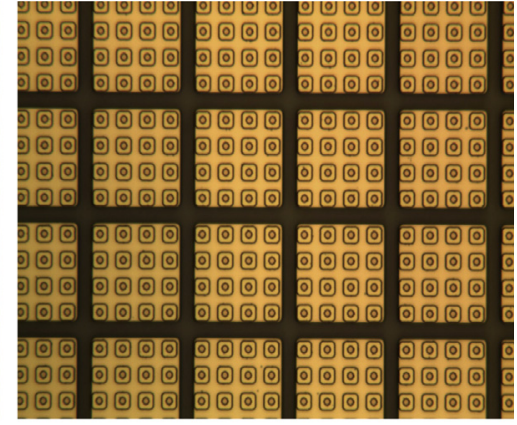

(B)

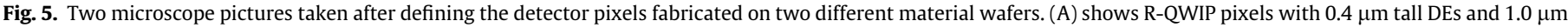
thick active material. (B) shows R-QWIP pixels with $0.45 \mu \mathrm{m}$ tall DEs and $1.2 \mu \mathrm{m}$ thick active material. Both bottom contact layers are $0.7 \mu \mathrm{m}$ thick.

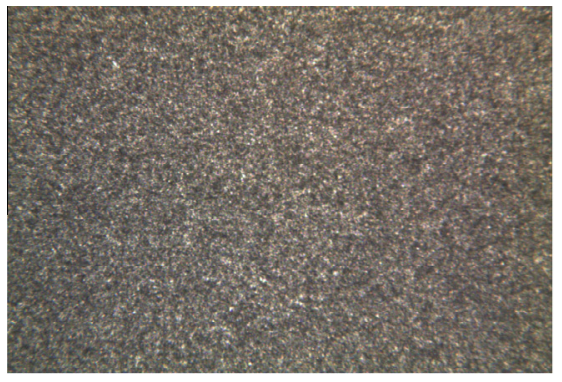

(A)

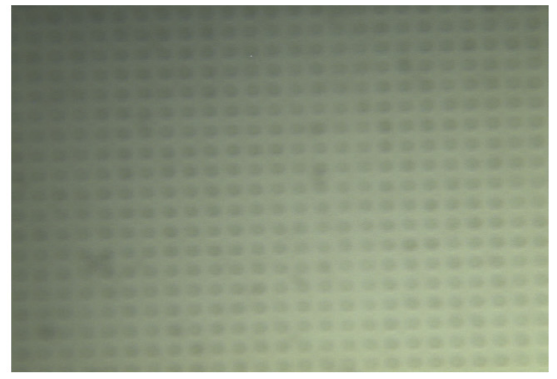

(B)

Fig. 6. The substrate surface (A) before and (B) after selective etching for substrate removal.

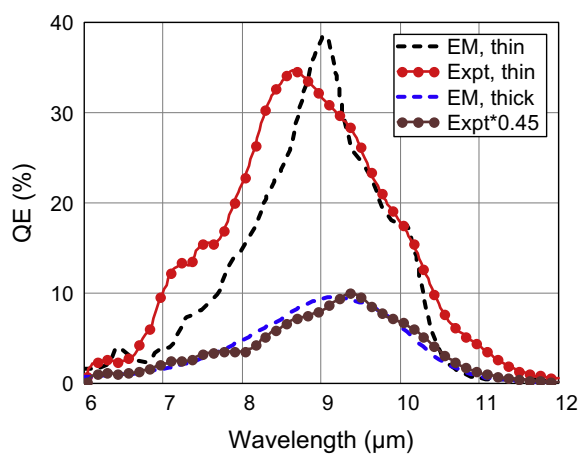

(A)

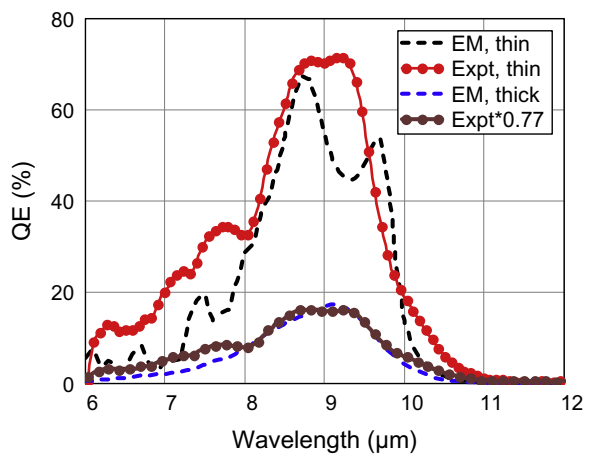

(B)

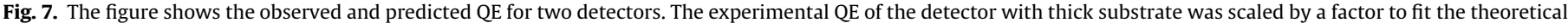
prediction.

photoconductive gain $\mathrm{g}$ at the voltage where $\mathrm{CE}$ saturates. The values of $\mathrm{QE}(=\mathrm{CE} / \mathrm{g})$ can then be calculated. Fig. 7 shows the experimental results for the two detectors. In general, the experimental results agree the predictions satisfyingly. For the detector in Figs. 5(A) and $7(\mathrm{~A})$, the calculated peak is at $9 \mu \mathrm{m}$ with $\mathrm{QE}=38.3 \%$. It is slightly larger than the observed $\mathrm{QE}=34.6 \%$ at $8.7 \mu \mathrm{m}$, which is deduced from $\mathrm{CE}=27.4 \%$ and $g=0.79$. For the detector in Figs. 5(B) and 7(B), the calculated peak is $67.3 \%$ at $8.6 \mu \mathrm{m}$, and the observed value is $70.8 \%$ at $8.8 \mu \mathrm{m}$, and $\mathrm{CE}=62.3 \%$ and $g=0.88$. Comparing with the thinned detectors, the detectors with thicker $(\sim 50 \mu \mathrm{m})$ substrates have significant lower QE as seen in Fig. 7. On the other hand, these measured thick substrate QEs are larger than that predicted by theory. We attribute the higher QE to the optical crosstalk among pixels, which is expected to be present in thick substrates.
The complete comprehensive performance test of first $\mathrm{R}$ QWIP was carried out in NASA/Goddard Space Flight Center. Fig. 8 shows the tested $\mathrm{CE}$ at multiple bias voltages and two detector temperatures. The highest CE is about $11 \%$. In addition, supplementary noise gain measurements from single pixel devices fabricated from the same wafer lot enabled the estimation of the QE distribution. The QE of the FPA is $29.4 \%$, which is slightly lower than predicted (33.1\%). The dark current limited detectivity is $1.76 \times 10^{11} \mathrm{~cm} \sqrt{ } \mathrm{Hz} / \mathrm{W}$ at $65 \mathrm{~K}$ and $4.39 \times 10^{11}$ $\mathrm{cm} \sqrt{ } \mathrm{Hz} / \mathrm{W}$ at $58 \mathrm{~K}$. The operability and spectral non-uniformity of the FPA are $99.57 \%$ and $3 \%$ respectively, where the operability is for NE $\Delta \mathrm{T}$ less than $25 \mathrm{mK}$ at $58 \mathrm{~K}$. The detail test results of the FPA will be published in a separate paper. Fig. 9 is an infrared image taken by the FPA. The test results of the test detectors and FPA indicate that no plasma damage to the detector 


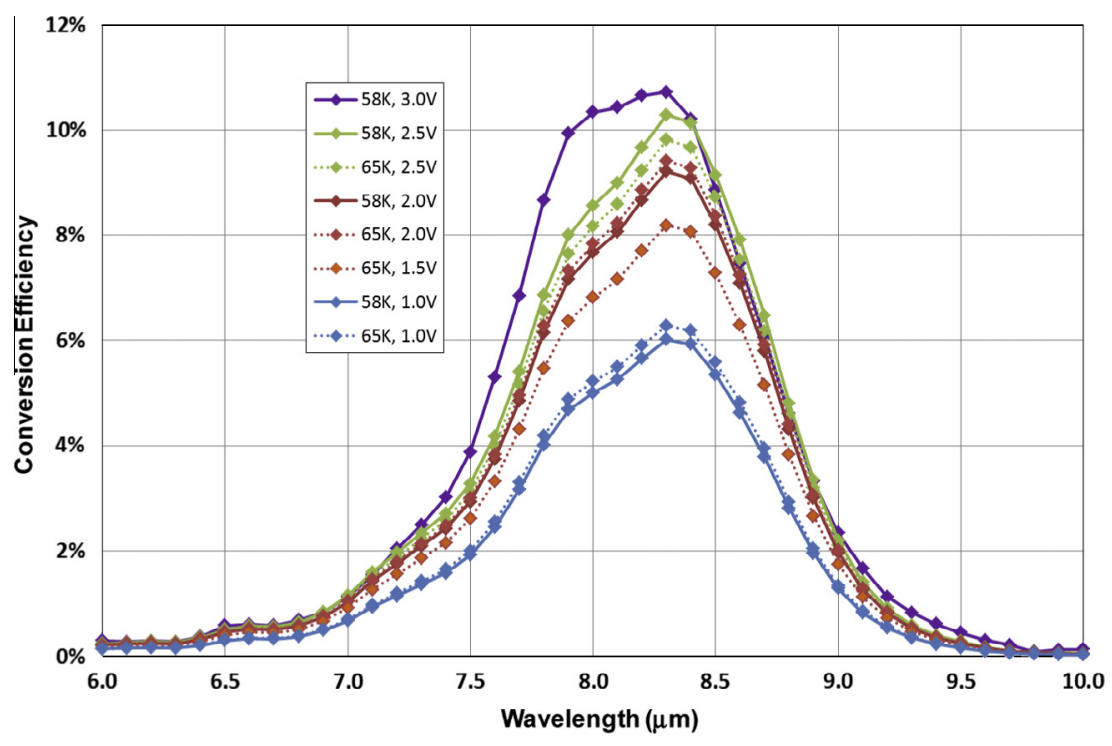

Fig. 8. The measured CE for multiple bias voltages and detector temperatures.

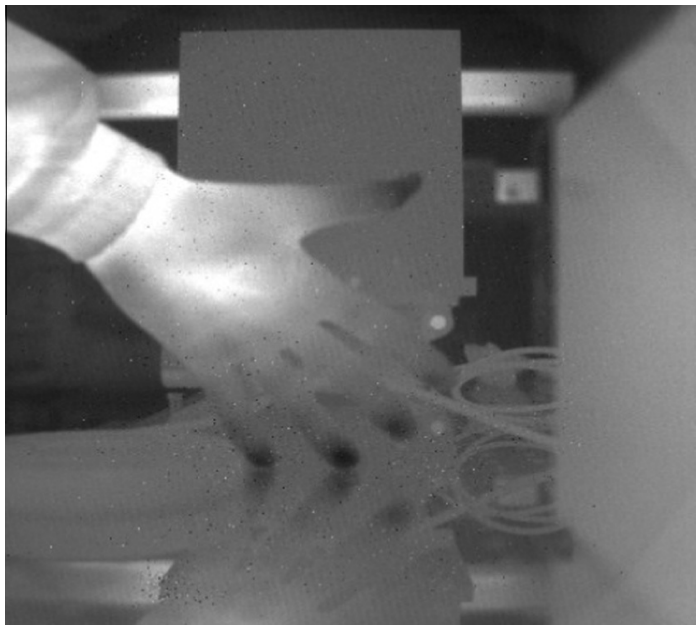

Fig. 9. An infrared image taken by first R-QWIP FPA.

material was observed in either wafer fabrication or substrate thinning.

\section{Conclusion}

Optimized selective and non-selective ICP etching processes were developed and applied to fabricate $40 \times 40$ small format test detector arrays and $640 \times 512$ and $1 \mathrm{~K} \times 1 \mathrm{~K}$ large format FPAs. Our selective ICP etching process has an optimized $\mathrm{BCl}_{3} / \mathrm{SF}_{6} / \mathrm{Ar}$ composition and shows a nearly infinite etching selectivity for the GaAs over the $\mathrm{Al}_{x} \mathrm{Ga}_{1-x} \mathrm{As}$ etch-stop layer. We used it to create the diffractive elements in the R-QWIP structures and remove the substrate completely. Meanwhile, the non-selective ICP etching process was used to perform straight sidewall, damage-free ground contact etching and pixel mesa etching. The test results of the R-QWIPs agree with EM designs satisfyingly. The highest QE observed are $70.8 \%$ and $29.4 \%$ for the test detector and first R-QWIP FPA correspondingly.

\section{Conflict of interest}

There is no conflict of interest.

\section{References}

[1] P. Bois, V. Guériaux, N. Brière de l'Isle, A. Manissadjian, H. Facoetti, X. Marcadet, E. Costard, A. Nedelcu, QWIP status and future trends at Thales, in: Proc. of SPIE 8268, 82682M1 - 11, 2012.

[2] S.U. Eker, Y. Arslan, C. Besikci, High speed QWIP FPAs on InP substrates, Inf. Phys. Technol. 54 (2011) 209-214.

[3] K.K. Choi, M.D. Jhabvala, D.P. Forrai, A. Waczynski, J. Sun, R. Jones, Electromagnetic modeling of quantum well infrared photodetectors, IEEE J. Quantum Electron. 48 (3) (March 2012) 384-393.

[4] K.K. Choi, Electromagnetic modeling of edge coupled quantum well infrared photodetectors, J. Appl. Phys. 111 (2012) 124507.

[5] K.K. Choi, M.D. Jhabvala, D.P. Forrai, A. Waczynski, J. Sun, R. Jones, Electromagnetic modeling and design of quantum well infrared photodetectors, IEEE J. Sel. Top. Quantum Electron. 19 (5) (2013) 3800310.

[6] K.K. Choi, M.D. Jhabvala, J. Sun, C.A. Jhabvala, A. Waczynski, K. Olver, Resonator-quantum well infrared photodetectors, Appl. Phys. Lett. 103 (2013) 201113.

[7] Jason Sun, K.K. Choi, Unchul Lee, Fabrication of Pyramidal corrugated quantum well infrared photodetector focal plane arrays by inductively coupled plasma etching with BCl3/Ar, J. Micro/Nanolithogr. MEMS MOEMS 11 (4) (2012) 043003.

[8] A. Mitchell, R.A. Gottscho, S.J. Pearton, G.R. Schetler, Real-time, in situ monitoring of GaAs and AlGaAs photoluminescence during plasma processing, Appl. Phys. Lett. 56 (9) (1990) 821-823.

[9] C.M. Knoedler, L. Osterling, H. Shtikman, Reactive ion etching damage to GaAs layers with etch stops, J. Vac. Sci. Technol. B6 (1988) 1573.

[10] S.J. Pearton, M.J. Vasile, K.S. Jones, K.T. Short, E. Lane, T.R. Followan, A.E. VonNeida, N.M. Haegel, Reactive ion etching of GaAs with $\mathrm{CCl}_{2} \mathrm{~F}_{2}: \mathrm{O}_{2}$ : etch rates, surface chemistry, and residual damage, J. Appl. Phys. 65 (1989) 12811292.

[11] T.R. Hayes, U.K. Chakrabarti, F.A. Baiocchi, A.B. Emerson, Damage to InP and InGaAsP surfaces resulting from $\mathrm{CH}_{4} / \mathrm{H}_{2}$ reactive ion etching, J. Appl. Phys. 68 (1990) 785.

[12] J. Etrillard, P. Ossart, G. Patriarche, M. Juhel, J.F. Bresse, C. Daguet, Anisotropic etching of InP with low sidewall and surface induced damage in inductively coupled plasma etching using $\mathrm{SiCl}_{4}$, J. Vac. Sci. Technol. A 15 (3) (1997) 626.

[13] J. Sun, K.K. Choi, M.D. Jhabvala, C. Jhabvala, Advanced substrate thinning process for GaAs-based devices, J. Micro/Nanolithogr. MEMS MOEMS 10 (2) (2011) 023004.

[14] S. Golka, M. Arens, M. Reetz, T. Kwapien, Time-multiplexed, inductively coupled plasma process with separate $\mathrm{SiCl}_{4}$ and $\mathrm{O}_{2}$ steps for etching of GaAs with high selectivity, J. Vac. Sci. Technol. B 27 (2009) 2270. 